\title{
CFTR Modulator Therapy and Its Impact on Lung Transplantation in Cystic Fibrosis
}

\author{
Christian Benden (D) - Carsten Schwarz
}

Received: July 4, 2021 / Accepted: August 3, 2021 / Published online: August 18, 2021

(C) The Author(s) 2021

\begin{abstract}
Cystic fibrosis (CF) is the most common autosomal recessive disorder in Caucasian people and is caused by mutations in the gene encoding for the $\mathrm{CF}$ transmembrane conductance regulator (CFTR) protein. It is a multisystem disorder; however, CF lung disease causes most of its morbidity and mortality. Although survival for CF has improved over time due to a multifaceted symptomatic management approach, CF remains a life-limiting disease. For individuals with progressive advanced CF lung disease (ACFLD), lung transplantation is considered the ultimate treatment option if compatible with goals of care. Since 2012, newer drugs, called CFTR modulators, have gradually become available, revolutionizing CF care, as these small-molecule drugs target the underlying defect in CF that causes decreased CFTR protein synthesis, function, or stability. Because of their extremely high efficacy and overall respectable tolerability, CFTR modulator drugs have already proven to have a substantial
\end{abstract}

\section{Benden ( $\square)$}

Faculty of Medicine, University of Zurich, Raemistrasse 71, 8006 Zurich, Switzerland e-mail: christian_benden@yahoo.de

C. Schwarz

Division of Cystic Fibrosis, CF Center

Westbrandenburg, Campus Potsdam, Potsdam,

Germany positive impact on the lives of individuals with CF. Individuals with ACFLD have generally been excluded from initial clinical trials. Now, however, these drugs are being used in clinical practice in selected individuals with ACFLD, showing promising results, although randomized controlled trial data for CFTR modulators in this subgroup of patients are lacking. Such data need to be gathered, ideally in randomized controlled trials including patients with ACFLD. Furthermore, the efficacy and tolerability of the newer modulator therapies in individuals with ACFLD need to be monitored, and their impact on lung disease progression and the need for lung transplantation as the ultimate therapy call for an objective evaluation in larger patient cohorts. As of today, guidelines for referral and listing of lung transplant candidates with $\mathrm{CF}$ have not incorporated the status of the new CFTR modulator therapies in the referral and listing process. The purpose of this review article, therefore, is threefold: first, to describe the effects of new therapies, with a focus on the subgroup of individuals with ACFLD; second, to provide an update on the recent outcomes after lung transplantation for individuals with CF; and third, to discuss the referral, evaluation, and timing for lung transplantation as the ultimate therapeutic option in view of the new treatments available in CF. 
Keywords: Advanced cystic fibrosis lung disease; CFTR modulator; Cystic fibrosis; Lung transplantation

\section{Key Summary Points}

Survival in individuals with cystic fibrosis (CF) has improved substantially, even before treating the underlying genetic defect encoding for the cystic fibrosis transmembrane conductance regulator (CFTR) protein.

CF lung disease causes most of the morbidity and mortality, with lung transplantation considered the ultimate treatment option for individuals with progressive advanced CF lung disease (ACFLD).

Since 2012, newer drugs called CFTR modulators have become available, revolutionizing $\mathrm{CF}$ care by targeting the underlying defect in CF that causes decreased CFTR protein synthesis, function, or stability.

Even though randomized controlled trial data for CFTR modulators in individuals with ACFLD are lacking, clinical usage of these drugs in selected individuals shows encouraging results, leading to removal of patients from transplant waiting lists and fewer lung transplants for performed for CF.

New consensus guidelines for the selection of CF candidates for lung transplantation should take the new therapies into consideration regarding timing of referral and listing for transplant.

\section{INTRODUCTION}

In the Caucasian population, cystic fibrosis (CF) is the most common autosomal recessive disorder, caused by mutations in the gene encoding for the CF transmembrane conductance regulator (CFTR) protein; the CFTR gene was discovered in 1989 [1]. Although CF is a multisystem disorder affecting tissues and organs where CFTR is expressed, CF lung disease causes most of the morbidity and mortality. In the past, most individuals with CF did not survive beyond childhood or adolescence due to progressive CF lung disease. However, survival of individuals with CF has expanded over time, not due to the repair of the underlying genetic defect, but through the combination of establishing CF registries for a better understanding of the natural history of $\mathrm{CF}$, the implementation of CF newborn screening in many countries, and the introduction of continuously improved symptomatic therapies [2]. The main pillars in tackling CF lung disease have traditionally been airway clearance techniques and antibiotic therapies. Newer therapies treating the downstream complications of CFTR dysfunction are under development, focusing on enhanced mucociliary clearance, improved delivery of antibiotics to the respiratory airways, and anti-inflammatory drugs [3]. Nevertheless, for individuals with progressive advanced $\mathrm{CF}$ lung disease (ACFLD), lung transplantation remains the ultimate treatment option that is nowadays considered the standard of care [4]. Comprehensive consensus guidelines were published by the Cystic Fibrosis Foundation (CF Foundation) in the United States (USA) for people with ACFLD, confirming that lung transplantation is an option for individuals with ACFLD if compatible with the overall goals of care; therefore, no individual with CF should die without consideration for lung transplantation [5]. In 2015, consensus recommendations on the appropriate timing for listing candidates with ACFLD for lung transplant were published by the International Society for Heart and Lung Transplantation (ISHLT) [6]. Although the overall survival benefit for lung transplantation in individuals with $\mathrm{CF}$ remains controversial within the CF and the lung transplant communities [7], recent data published in the ISHLT Thoracic Transplant Registry Report demonstrate increased median survival of more than 9 years following lung transplantation for CF, far superior to that for all other primary 
underlying diseases leading to lung transplantation [8].

In the meantime, newer drugs, so-called CFTR modulators, have gradually become available since 2012, revolutionizing CF care [3]. Nowadays, CF has become a disease "with a new face" through the introduction of these small-molecule drugs into clinical practice to target the underlying defect in CF that causes decreased CFTR protein synthesis, function, or stability $[2,9]$. To date, four orally administered drugs have been licensed by medical licensing agencies in Australia, Europe, and North America for clinical use in people with CF depending on the underlying genetic defect: ivacaftor $\left(\right.$ Kalydeco $\left.^{\circledR}\right)$, lumacaftor/ivacaftor $\left(\right.$ Orkambi $\left.^{\circledR}\right)$, tezacaftor/ivacaftor $\left(\right.$ Symdeko ${ }^{\circledR}$ or Symkevi ${ }^{\circledR}$ ), and the triple combination of elexacaftor/tezacaftor/ivacaftor $\left(\right.$ Trikafta $\left.^{\circledR}\right)$. Approximately $85 \%$ of individuals with CF could benefit from these CFTR modulator therapies, and based on the extremely high efficacy and the overall respectable tolerability of the triple-combination CFTR modulator drug, a substantial positive impact on the lives of individuals with $\mathrm{CF}$ can be expected [2]. As individuals with ACFLD have generally been excluded from clinical trials, it seems vital to monitor the efficacy and tolerability of the newer CFTR modulator therapies in this subgroup of individuals with CF and the impact on lung disease progression and the need for lung transplantation as the ultimate therapy in this scenario for individuals with CF, but also for the field of lung transplantation overall. To date, guidelines for referral and listing of lung transplant candidates with CF have not incorporated the status of the new CFTR modulator therapies in the referral and listing process [6].

Thus, the aim of this narrative review is threefold: first, to describe the effects of new therapies, with a focus on the subgroup of individuals with ACFLD; second, to provide an update on recent outcomes of lung transplantation for individuals with CF; and third, to discuss referral and evaluation for lung transplantation as the ultimate therapeutic option in view of the new treatments available for individuals with $\mathrm{CF}$.
This article is based on reviews of current consensus statements and published literature; it does not involve any studies with human participants or animals conducted by any of the authors. It fully complies with ethics guidelines.

\section{CFTR DYSFUNCTION IN CF}

Briefly, CF is caused by biallelic mutations in the CFTR gene [10]. CFTR is a transmembrane ion channel that regulates the transport of anions such as chloride and bicarbonate across epithelial cell membranes $[10,11]$. In $C F$, the decreased function and/or quantity of CFTR protein leads to aberrant epithelial electrolyte transport in different organs and tissues, resulting in multisystemic disease involving progressive loss of lung function, pancreatic insufficiency, and liver disease [10, 11]. More than 2000 CFTR mutations are known, but Phe508del is the most common CF-causing CFTR allele $[10,12]$. Nearly $90 \%$ of people with CF have $\geq 1$ Phe508del allele [10, 12, 13].

CFTR mutations can be divided into six classes according to the molecular phenotype of the CFTR protein [14-16]. Class I mutations are mostly deletions, stop codon sequences with a reading frame shift, which cause a disturbance in protein synthesis. This leads to a break in translation, and no complete protein strand is formed. Class II mutations lead to misfolding of the protein, which is then prematurely degraded by the proteasome. The consequence in both cases is reduced or absent transport of the CFTR to the cell membrane. As a result, with class I and II mutations, there is almost no CFTR found at the cell membrane. Class III mutations lead to a reduced opening probability due to a defective regulation of the CFTR function ("gating"). The first CFTR mutation for which the CFTR modulator ivacaftor was approved is the G551D gating mutation. Class IV mutations are associated with decreased conductance of the CFTR, resulting in reduced chloride ion flux. Class $\mathrm{V}$ mutations result in a decreased amount of functional CFTR protein, mostly CFTR mutations with alternative splicing, defects in the promoter region, or mutations localized in the intron. A proportion of wild-type CFTR is 
often still present. Class VI mutations are characterized by reduced stability. Although there is a normal amount in the cell membrane, they are subject to premature lysosomal degradation [14-16]. The most common mutation in the Caucasian population, Phe508del, is characterized as a class II mutation. Because of a deletion of the amino acid phenylalanine, the cytoplasmic and membrane-integrated domains cannot be attached, and the maturation of the protein is impaired. However, some of the defective CFTR molecules reach the cell membrane, where their functionality is impaired by the defective tertiary structure; in addition, membrane stability is reduced due to premature endocytosis. Thus, Phe508del can be classified primarily as a class II mutation, but secondarily as a mutation of classes III and VI. Class I-III mutations result in a majority of negligible CFTR residual function of a maximum of $10 \%$ and can therefore be described as severe mutations. Mutations of classes IV-VI are mostly mild mutations, which are associated with a clinically relevant residual CFTR function [14-16].

\section{TARGETED THERAPIES AIMING TO RESTORE CFTR FUNCTION}

Only symptomatic therapeutic approaches have been applied in the past decades, but studying and defining the cellular and molecular pathology of CFTR mutations have been the first steps and prerequisites for the development of small-molecule compounds targeting the underlying defect in CF. The knowledge that some CFTR variants carrying class III or IV mutations are expressed at the apical membrane of secretory epithelial cells at a density similar to that of the wild-type protein was the basis for the development of gating potentiators that are able to increase the probability of opening, resulting in increased plasma membrane chloride conductance [17]. The first targeted CFTR mutation was G551D, for which VX-770 (ivacaftor) was the first potentiator drug to be approved for CF treatment by the licensing authorities in the USA. Ivacaftor directly targets the gating defect of the class III mutation
G551D-CFTR [18]. It is no exaggeration to say that ivacaftor has ushered in a new era of CF treatment. Ivacaftor was developed by a pharmaceutical company in conjunction with Cystic Fibrosis Foundation Therapeutics, Inc. The compound demonstrated remarkable clinical benefit in patients carrying the G551D mutation in either one or two alleles [18-20]. The approval of VX-770 was then extended to gating mutations other than G551D [21-23]. In addition, several novel potentiators are currently under experimental and/or clinical investigation and are demonstrating promising effects as well [24-26]. VX-561 (deutivacaftor or formerly CTP-656; Vertex Pharmaceuticals), a potentiator, is a deuterated form of ivacaftor that has shown enhanced stability in vitro and in healthy volunteers compared to ivacaftor [27]. The benefit for patients would be to allow people with CF to take it once daily rather than twice, as is the case with ivacaftor. The prototypical class II mutation, Phe508del, is defined by a complex folding defect that compromises both NBD1 stability and the channel's cooperative domain assembly [21, 22]. With this knowledge, the goal was to find correctors that act as pharmacological chaperones, so that direct binding would be possible while also promoting the biogenesis of class II CFTR mutations. Pharmacological chaperones can be classified in terms of their molecular targets in the CFTR structure: class 1 correctors have the potential to stabilize NBD1-TMD1 and/or NBD1-TMD2 interfaces; class 2 correctors stabilize NBD2 and its interfaces with other CFTR domains; and class 3 correctors directly stabilize NBD1 [28]. With this new classification, further research is feasible to develop new or adapted correctors or combinations of correctors.

The first highly promising corrector compound was VX-809 (lumacaftor). This compound stabilizes the NBD1-MSD1/2 interface by reverting the PheF508 CFTR functional expression defect. The licensing authorities in Europe and the USA approved co-treatment with lumacaftor/ivacaftor (Orkambi ${ }^{\circledR}$ ) for Phe508delhomozygous patients aged $\geq 12$ years in 2015 [29]. In many countries, lumacaftor/ivacaftor is now approved from the age of 2 years. A secondgeneration corrector is tezacaftor (VX-661; 
Vertex Pharmaceuticals). It has been developed to possess better pharmacokinetic properties and fewer adverse effects than lumacaftor/ivacaftor. Tezacaftor/ivacaftor studies resulted in comparable clinical outcomes to those with lumacaftor/ivacaftor in patients who were homozygous for the Phe508del mutation $[30,31]$. Interestingly, co-treatment with tezacaftor/ivacaftor was more effective in patients who carried the Phe508del-heterozygous with a residual function mutation in trans [32]. Thus, in 2018, the licensing authorities in Europe and the USA approved co-treatment with tezacaftor/ ivacaftor (Symdeko ${ }^{\circledR}$ or Symkevi ${ }^{\circledR}$ ) for patients aged $\geq 12$ years who were Phe508del-homozygous or Phe508del-heterozygous with a residual function mutation in trans, which was later extended in the USA for patients aged $\geq 6$ years. As the dual combinations lumacaftor/ivacaftor and tezacaftor/ivacaftor were far below expectations in terms of clinical response and demonstrated only modest efficacy in Phe508del-homozygous patients, new compounds have since been tested. Four novel correctors, VX-152, VX-440, VX-445, and VX-659, have been found to improve CFTR activity when coadministered with tezacaftor/ivacaftor in human bronchial epithelial cells (Phe508del/ Phe508del genotype), and two compounds (VX445 [or elexacaftor] and VX-659 [or bamocaftor]) were selected for clinical studies and demonstrated more suitable pharmacological properties and safety profiles for long-term use. Both triple combinations tezacaftor/ivacaftor/ VX-445 and tezacaftor/ivacaftor/VX-659 were safe, with most adverse effects being mild to moderate, and led to a reduction in sweat chloride concentration and bronchopulmonary exacerbation frequency in phase II and III trials. In addition, a significant increase in predicted forced expiratory volume in one second $\left(\mathrm{FEV}_{1}\right)$ was also achieved after triple-combination therapy in Phe508del-homozygous patients (up to $11.0 \%$ for the combination with VX-445 and $9.7 \%$ for the combination with VX-659 compared to tezacaftor/ivacaftor only) and Phe508del-heterozygous patients with a minimal function mutation in trans (up to $14.3 \%$ for the combination with VX-445 and $13.3 \%$ for the combination with VX-659 compared to placebo) [30, 33-36].

\section{USE OF CFTR MODULATORS IN CLINICAL PRACTICE}

Ivacaftor, lumacaftor/ivacaftor, tezacaftor/ivacaftor, and elexacaftor/tezacaftor/ivacaftor are drugs approved by licensing authorities in Europe and the USA, but the availability of these drugs depends on national health authorities and their decisions regarding reimbursement. Although no general guidelines for their usage exist, in Germany, a group of experts have started to develop a CFTR modulator therapy guideline that will hopefully provide clinicians guidance in treating individuals with CF using CFTR modulators [37]. Real-world data from different studies confirm the results from phase II and III trials discussed above [38-41]. In addition, long-term data are very promising in terms of exacerbation rate and loss of lung function, in particular for ivacaftor [41]. Analysis of registry data from both the United Kingdom (UK) and the USA has demonstrated that predicted $\mathrm{FEV}_{1}$ and exacerbation rate were significantly improved in the ivacaftor group relative to the comparator after 4 and 5 years of treatment, respectively. Most strikingly, detection of Pseudomonas aeruginosa in respiratory samples decreased within 4 and 5 years of treatment, respectively, but in the comparator group, an increase in Pseudomonas aeruginosa was detected [42].

\section{CFTR MODULATOR DEVELOPMENT PIPELINE AND FORECASTING STUDIES}

Several novel potentiators and correctors are under development by pharmaceutical companies. In addition to the previously mentioned VX-561 (deuterated form of ivacaftor), the molecules FDL176 (Flatley Discovery Lab) and PTI-808 (Proteostasis Therapeutics) are potentiators currently under evaluation [43]. Further, FDL169 (Flatley Discovery Lab) has demonstrated rescue of CFTR PM expression in 
F508del-expressing cells with similar efficacy as lumacaftor, but it is currently not under clinical evaluation. The molecules ABBV-2222, ABBV2737, ABBV-2851, ABBV-3221, and ABBV-3748 (developed by AbbVie-Galapagos) are very promising corrector drugs and comparable to lumacaftor or tezacaftor, but these molecules may be even more potent. In addition, PTI-801 (a third-generation corrector, developed by Proteostasis Therapeutics) showed good activity in vitro and also in a phase I trial, with significantly improved predicted $\mathrm{FEV}_{1}$, body mass index (BMI), and decreased sweat chloride concentration in people with CF who were Phe508del-homozygous and received tezacaftor and ivacaftor [43]. In addition to correctors and potentiators, antisense oligonucleotides (QR010, ProQR) for correcting aberrant splicing, amplifiers to increase the abundance of protein substrate (PTI-428, Proteostasis Therapeutics), and read-through agents and nonsense-mediated mRNA decay inhibitors to rescue protein synthesis (NB124, Eloxx Pharmaceuticals, PTC124 and Translarna ${ }^{\circledR}$, PTC Therapeutics) are presently being evaluated regarding their impact on clinical outcome in people with CF [43].

Several clinical trials of treatments aimed at significantly modulating the CFTR defect in people with CF are starting in phase I or II [44]. These include MRT5005, a new drug designed to restore CFTR function by delivering correct copies of CFTR-encoded mRNA to the lungs, and ABBV-191, a type of CFTR potentiator. The following studies are in phase II: VX-561 (altered form of the potentiator ivacaftor), VX-121 (corrector), ELX-02 (restore CFTR function), ABBV-3067 (potentiator), and ABBV-2222 (corrector). As Phe508del is the most common CFTR mutation in CF worldwide, studies have focused on this patient population. But approximately $20 \%$ of people with CF have rare, ultrarare, or even unique CFTR mutations. To study drugs in " $n=1$ " studies, correlations between in vitro or ex vivo tests and various clinical parameters are crucial. For example, pairing in vitro measurement of CFTR function in cell lines and clinical features showed a significant correlation between CFTR function and sweat chloride concentration, lung function, and pancreatic status [45]. Correlations between responses in patient-derived specimens and clinical parameters/biomarkers have been investigated to establish reliable prediction of drug effectiveness. A consistent correlation was found among forskolin-induced swelling of intestinal organoids, sweat chloride concentration, and intestinal current measurements of infants with CF [46]. In summary, huge strides have been made and highly effective modulators are available for many people with $\mathrm{CF}$, but additional drug development is still needed to help the entire population with CF.

\section{IMPACT OF CFTR MODULATOR THERAPY ON OUTCOMES IN PEOPLE WITH ACFLD}

Although there have been remarkable steps forward in recent years in terms of survival and quality of life in $\mathrm{CF}$, a significant number of patients-in particular adults-with CF continue to experience ACFLD [5]. As this subgroup of patients is at higher risk for complications and has a limited life expectancy, as well as usually a lower quality of life, effective treatments are of high interest in this subpopulation. However, pharmacological studies usually exclude this subgroup of patients, as less clinical response is expected in comparison to patients with better lung function tests. Fortunately, there are some approaches, mainly investigatorinitiated trials, with a focus on real-world experiences, to answer the question of whether CFTR modulators may influence clinical outcomes in ACFLD. Only two studies, one from Ireland and the UK, and one from Italy, have evaluated the outcome in adults with CF and predicted $\mathrm{FEV}_{1}<40 \%$ who received ivacaftor within a compassionate use program $[47,48]$. In the first study, ivacaftor was clinically effective in 21 patients with ACFLD who carried the G551D mutation; $\mathrm{FEV}_{1}$ improved from 26.5 to $30.7 \%(p=0.01)$, which represented a $16.7 \%$ relative improvement after treatment with ivacaftor. In addition, median BMI improved $\left(49.8-51.6 \mathrm{~kg} / \mathrm{m}^{2}, \quad p=0.006\right)$ as well. Most striking was that the median length of inpatient intravenous antibiotic treatment decreased 
from 23 to 0 days/year $(p=0.001)$. The second study revealed a clinical benefit in 13 patients with ACFLD; predicted $\mathrm{FEV}_{1}$ increased from 35.1 to $46.6 \%$ after 12 months of treatment with ivacaftor (absolute increase $11.5 \%$ and relative increase $32.8 \%$ ). Further, there was a significant decrease in pulmonary exacerbations $(p=0.0048)$, increase in BMI $(p=0.0031)$, and decrease in sweat chloride concentration $(p<0.0001)$. No major safety concerns were found in either study, supporting the good results in this specific patient subgroup. The best evaluation was in patients with ACFLD and Phe508del homozygous mutation and treatment with lumacaftor/ivacaftor. Significant improvement in predicted $\mathrm{FEV}_{1}$ was demonstrated in a subgroup analysis of a pooled data set of the TRANSPORT and TRAFFIC phase III studies [49]. In total, 1108 patients were included in the entire study, $7 \%(n=81)$ with a predicted $\mathrm{FEV}_{1}$ value that decreased to $<40 \%$ between the screening and the baseline visits. In addition to $\mathrm{FEV}_{1}$ improvement, BMI improved significantly; although treatment was generally well tolerated, the incidence of some respiratory adverse events was higher with lumacaftor/ivacaftor than with placebo in all subgroups [49]. In patients with baseline predicted $\mathrm{FEV}_{1}$ values $<40 \%$, these adverse events included cough, dyspnea, and abnormal respiration [49]. Real-world studies revealed different results; however, these studies usually included only small numbers of patients with ACFLD. A Danish study with 21 ACFLD patients demonstrated significant improvement in predicted $\mathrm{FEV}_{1}$ but not in BMI [50]. Nevertheless, health-related quality of life showed a significant overall improvement. The French experience also revealed a predicted $\mathrm{FEV}_{1}$ increase, but no increase in BMI; unfortunately, $30 \%$ of patients in this multicenter real-world trial discontinued the medication due to respiratory side effects [51]. A small Australian study evaluated 11 patients with ACFLD and revealed that all study patients had a $\mathrm{FEV}_{1}$ decrease after 1 month, which was also the end of the observation period [52]. As respiratory adverse events have frequently been reported, investigators in the USA divided their 46 study patients with ACFLD into patients with a full dose and a half dose of lumacaftor/ivacaftor, respectively [53]. The investigators reported no change in predicted $\mathrm{FEV}_{1}$ or BMI, but a lower hospitalization rate and duration of intravenous antibiotics. The study group on the half lumacaftor/ivacaftor dose generally tolerated the drug better, and therefore, the authors recommend starting patients with ACFLD on a reduced drug dose before increasing to full dose [53]. Like the French experience, an Australian multicenter case-control study revealed frequent adverse events, including chest tightness and dyspnea, in $55 \%$ of patients, resulting in drug termination in $32 \%$ [54]. Another Australian study produced very promising results in patients with ACFLD $(n=40)$ [55]. The study results showed significantly fewer hospital days, lower exacerbation rates, improvements in predicted $\mathrm{FEV}_{1}$ and BMI, and fewer days on intravenous antibiotic [55]. In a Swiss study, ten patients with ACFLD who commenced treatment with lumacaftor/ivacaftor showed no significant change in $\mathrm{FEV}_{1}$, BMI, or exacerbation rate, but two patients were ultimately paused on the lung transplant waiting list, an important achievement [56]. Stabilization of lung function and BMI may be regarded as crucial in patients with progressive ACFLD, as they usually experience a decline in lung function and decreased BMI due to recurrent pulmonary infections.

The documented high frequency of adverse events in patients receiving lumacaftor/ivacaftor led to the conductance of a recent phase III study including patients with CF who discontinued lumacaftor/ivacaftor due to respiratory events [57]. In this phase III study, patients with ACFLD were also allowed to participate. The primary study endpoint was incidence of predefined respiratory adverse events of particular interest (chest discomfort, dyspnea, abnormal respiration, asthma, bronchial hyperreactivity, bronchospasm, wheezing). Respiratory adverse events were significantly lower in the tezacaftor/ivacaftor subgroup than in the placebo group (14.0\% vs. $21.3 \%)$ [57]. The adverse events were mild or moderate in severity, and no treatment disruption or discontinuation occurred. The mean predicted $\mathrm{FEV}_{1}$ at baseline was $44.6 \% \pm 16.1 \%$ in the tezacaftor/ivacaftor group and $48.0 \% \pm 18.1 \%$ 
in the placebo group. The posterior mean difference in absolute change in predicted $\mathrm{FEV}_{1}$ from baseline to the average value of days 28 and 56 was 2.7 percentage points for patients on tezacaftor/ivacaftor treatment versus placebo [57]. These data indicate substantial clinical improvement in multiple dimensions, although relevant adverse events potentially occur. As patients with ACFLD are the subgroup of patients with the greatest need for efficient treatments, the authors believe that the advantages of CFTR modulator therapy outweigh the disadvantages in this specific patient subgroup with ACFLD.

O'Shea and colleagues from Dublin, Ireland, recently published a case series of 14 adults with ACFLD all receiving elexacaftor/tezacaftor/ivacaftor as part of a managed access program [58]. The mean predicted $\mathrm{FEV}_{1}$ in patients prior to start of the triple CFTR modulator therapy was $27 \pm 7.3 \%$, and $64 \%$ had CF-related diabetes; patients had a mean BMI of $20.7 \pm 3.6 \mathrm{~kg} / \mathrm{m}^{2}$ and a median of three hospitalizations in the year before starting the new drug [58]. All measured clinical parameters improved significantly during this observational study, with a mean \pm SD follow-up of $4.9 \pm 1.94$ months. Although these are single-center data with unblinded drug administration and a potential selection bias, they demonstrate that elexacaftor/tezacaftor/ivacaftor is highly effective in improving multiple clinical parameters, comparable to effects seen in individuals with milder CF [58].

\section{LUNG TRANSPLANTATION AS STANDARD OF CARE IN CF}

In 1988, the first successful double-lung transplantation for CF was carried out by Joel D. Cooper and colleagues in Toronto, Canada [59]. Just over three decades later, lung transplantation is considered an accepted therapeutic option children and adults with progressive ACFLD if compatible with goals of care $[4,60]$. If transplant candidates are optimally selected, a net survival benefit and improved health-related quality of life can be expected [4, 61-63]. Historically, CF is the third most common primary indication for lung transplantation in adults, following chronic obstructive pulmonary disease and idiopathic interstitial pneumonia [8]. In children and adolescents undergoing lung transplantation, CF is overall the most common underlying disease, reaching $67 \%$ in children over the age of 11 years [64].

The ISHLT Pulmonary Council published consensus recommendations for the selection of candidates for lung transplantation in 2015, including disease-specific considerations for $\mathrm{CF}$ [6]. Briefly, in candidates with $\mathrm{CF}$, referral should be considered if the $\mathrm{FEV}_{1}$ has fallen to $30 \%$ or patients with ACFLD show a rapid decline despite optimal medical therapy (particularly female patients), a 6-minute walk test distance $<400 \mathrm{~m}$, pulmonary hypertension outside hypoxic exacerbation, or any clinical deterioration associated with any of the following: acute respiratory failure requiring noninvasive ventilation, worsening nutritional status even with optimal nutritional intake and supplementation, pneumothorax, life-threatening hemoptysis despite bronchial embolization, and an increasing antibiotic resistance and poor clinical recovery from exacerbations [6]. Specific airway pathogens such as Burkholderia cenocepacia, Burkholderia gladioli, and multidrugresistant Mycobacterium abscessus require particular attention, as these pathogens are considered a relative contraindication by some transplant centers; thus, transplant candidate evaluation at centers that have substantial experience with such pathogens and their periand post-transplant management is recommended [6]. However, the general presence of pan-drug-resistant organisms in patients with CF does not seem to negatively affect 3- and 12-month post-transplant mortality according to a recent study using ISHLT Thoracic Transplant Registry data for 697 lung transplant recipients with $\mathrm{CF}$ with pan-resistant infection compared to 2649 controls [65]. Although increased infection-related mortality was shown, it was not predictive of mortality in the multivariate analysis [65]. Nevertheless, the study authors highlight the need for very attentive follow-up of lung transplant recipients with pan-resistant infections [65]. 
According to Weill et al., candidates with CF should be listed for lung transplantation in the case of chronic respiratory failure with hypoxia alone (partial pressure of oxygen $<8 \mathrm{kPa}$ or $<60 \mathrm{mmHg}$ ) or with hypercapnia (partial pressure of carbon dioxide $>6.6 \mathrm{kPa}$ or $>50 \mathrm{mmHg}$ ), long-term noninvasive ventilatory support, pulmonary hypertension, frequent hospitalization, rapid lung function decline, or WHO functional class IV symptoms [6]. For late referrals or prolonged waiting list times and deteriorating clinical status, extracorporeal life support should be considered as a "bridge" to lung transplant in highly select candidates of young age, absence of multi-organ dysfunction, and an appropriate potential for postoperative rehabilitation [6]. Ideally, candidates considered for a bridge to lung transplant should already be fully evaluated and listed for transplant, as a thorough multidisciplinary transplant assessment takes time. Preoperatively, extracorporeal life support systems are nowadays well established for cardiopulmonary bridge to lung transplant, even in pediatric lung transplant candidates, at experienced transplant centers [66-70]. But extracorporeal life support as a bridge strategy to lung transplantation is only advised in patients with a rapidly deteriorating condition with severe hypoxemia and/or hypercapnia, severe pulmonary hypertension, and/or right ventricular failure, until a suitable donor organ becomes available. Today, pretransplant extracorporeal life support is considered a superior alternative to long-term mechanical ventilation [70]. If possible, patients on extracorporeal life support as bridge to lung transplantation should be kept awake, even in the pediatric setting [60, 71-73]. To avoid late referrals for lung transplant necessitating the use of bridging strategies to transplant, early referral to a transplant center is encouraged. The US CF Foundation recently set out earlier thresholds for lung transplant referral in a consensus guideline document [74]. For adults with $\mathrm{CF}$, the CF Foundation recommends lung transplant referral no later than $\mathrm{FEV}_{1}<50 \%$ predicted and rapidly falling, or $\mathrm{FEV}_{1}<40 \%$ predicted with markers of shortened survival (6minute walk test distance $<400 \mathrm{~m}$, hypoxemia at rest or with exertion, hypercarbia, pulmonary hypertension), or FEV1 $<30 \%$ predicted [74]. In individuals with $\mathrm{CF}<18$ years of age, the $\mathrm{CF}$ Foundation recommends lung transplant referral no later than $\mathrm{FEV}_{1}<50 \%$ predicted and rapidly declining $\mathrm{FEV}_{1}<50 \%$ predicted with markers of shortened survival (as listed above), or FEV $1<40 \%$ predicted [74]. Currently, updated consensus recommendations by the ISHLT Pulmonary Council for the selection of adult and pediatric lung transplant candidates are under review, including considerations for individuals with $\mathrm{CF}$, with publication expected in 2021. At present, existing consensus guidelines for the candidate selection do not take the clinical effectiveness of the new CFTR modulator therapies and their use in daily clinical practice into consideration with regard to recommended thresholds for transplant referral and transplant listing. Bermingham et al. very recently published data on a short-term retrospective cohort study from three US centers including 50 adults with ACFLD on elexacaftor/ tezacaftor/ivacaftor, investigating its potential impact on lung transplant planning based on existing CFF guidelines [75]. For this purpose, patients were theoretically subcategorized as (1) "meeting indication for transplant center referral," (2) "annual transplant-focused discussion," or (3) "no transplant planning indicated" [75]. The study authors showed that prior to the start of triple-combination CFTR modulator therapy, all study patients fulfilled criteria for either annual transplant-focused discussion or transplant center referral. Conversely, after the start of triple-combination CFTR modulator therapy, seven of 50 patients were re-categorized as no transplant planning indicated, due to substantial lung function improvement, and 19 fewer patients fulfilled criteria for transplant center referral [75]. However, it is important to note that despite the high clinical effectiveness of CFTR modulator therapies even in patients with ACFLD, randomized controlled trial data in this subgroup are lacking, particularly regarding long-term durability and potential adverse clinical effects [76, 77]. Furthermore, there are individuals with CF who are not eligible for this regimen due to an underlying genetic defect, severe drug-related side effects, or lack of clinical response to CFTR modulators. As the CF and 
lung transplant community learns more about the impact of the new CFTR modulator therapies on long-term outcomes in individuals with $\mathrm{CF}$, currently recommended thresholds for transplant referral and transplant listing will require reassessment.

General contraindications for lung transplantation are described in detail by Weill and co-authors [6]. Briefly, absolute contraindications include a recent history of malignancy, uncorrected coronary artery disease or confirmed end-organ ischemia, acute sepsis, gross obesity, uncorrectable bleeding diathesis, substantial chest wall or spinal deformity, poorly controlled chronic pre-transplant infection with highly virulent and/or resistant pathogens, active Mycobacterium tuberculosis infection, and multiple organ failure, unless a combined organ transplant is an option (e.g., combined liver-lung transplantation in an individual with progressive ACFLD and deteriorating liver function) [6]. Further, substantial non-adherence to medical therapy or a psychiatric/psychological condition resulting in an inability to adhere to medical therapy or a lack of adequate of reliable social support could be considered an absolute contraindication; however, such circumstances require a sound multidisciplinary assessment and decision. Relative contraindications differ among transplant centers, and it is advisable to get in touch with the transplant center early in the referral process to discuss these. As CF is a multisystem disorder, extrapulmonary disease manifestations must be evaluated carefully as part of the lung transplant assessment.

A discussion of CF-specific intraoperative surgical considerations is beyond the scope of this review. CF lung transplant recipients need specialized postoperative care, as CF is a multisystem disorder, and CF lung transplant recipients have unique comorbidities [78]. The US CF Foundation very recently published a consensus document providing such recommendations to lung transplant and CF physicians for perioperative care and management of CF-specific comorbidities post transplant [78]. The authors acknowledge that clinical care teams even of large-volume lung transplant programs may well have less experience in the perioperative management of CF-associated comorbidities, such as CF-related diabetes mellitus, malabsorption, CF-related bone disease, and CF-related sinus disease, and particularly the perioperative management of CF-specific pathogens [78]. Hayes et al. demonstrated that transplant center volume was not associated with survival 1-year post lung transplant, based on United Network for Organ Sharing data; however, CF-specific knowledge predicted enhanced long-term outcomes for CF lung transplant recipients. On the other hand, general transplant knowledge was not associated with post-transplant survival in individuals with CF [79]. In general, if lung transplant candidates with CF are carefully selected, lung transplantation as the ultimate therapeutic option maximizes overall patient survival and provides improved health-related quality of life [4]. The overall post-transplant survival in individuals with $\mathrm{CF}$ has increased in recent decades to a median of more than 9 years according to ISHLT Thoracic Transplant Registry figures, superior to all other primary indications for lung transplantation [8]. Also, in children with $\mathrm{CF}$, overall survival following lung transplantation is nowadays comparable to that in adults [60, 64]. However, adolescent lung transplant recipients-predominantly individuals with $\mathrm{CF}$-have poorer overall survival than younger children and adults, with 15- to 19-year-old patients experiencing the highest risk of death $[60,63,80]$. Median survival in pediatric lung transplant recipients was 7 years for patients undergoing lung transplantation during the period from 2008 to 2016 according to the ISHLT Thoracic Transplant Registry report [81]. In recent years, the frequency and proportion of adults with $\mathrm{CF}$ undergoing lung transplantation has increased relative to that in children [82]. Benden and colleagues analyzed these trends over time, and examined post-transplant outcome and morbidity in CF lung transplant recipients using ISHLT Thoracic Transplant Registry data comprising more than 5000 patients [82]. The authors showed that post-transplant survival has recently increased, and so has the mean patient age; thus, a growing number of adults with CF currently receive lung transplants [82]. 
Further, adults with CF after lung transplantation have superior outcomes compared to children, and amongst adults, younger patients have inferior outcomes compared to older individuals [82]. To date, there are limited data on the impact of the new, highly effective CFTR modulator therapies on the number of lung transplants performed in individuals with CF. A very recent French study collecting national data on lung transplant activity from December 2019 to August 2020 revealed a twofold decline in the number of lung transplant procedures for CF compared with the previous 2 years [83]. Although these are preliminary data covering lung transplant activity only in France over a short time period, the impact of the new CFTR modulator therapies seems substantial. Nevertheless, additional multinational data are needed to more thoroughly evaluate the long-term impact of the newer treatments on lung transplant volumes. Overall, early post-transplant complications include primary graft dysfunction and cytomegalovirus and other infections. The latter remains a particular issue in lung transplant recipients with CF due to the chronic pulmonary infection with typical CF pathogens pre-transplant, including the upper airways in individuals with known chronic CF-related sinus disease. The major obstacle for extended post-transplant survival remains the development of chronic lung allograft dysfunction (CLAD), which affects pediatric and adult lung transplant recipients alike $[8,64]$. Bronchiolitis obliterans syndrome (BOS), the most common form of CLAD, is the primary cause of death at 5 years after lung transplantation [8]. Clinical practice guidelines for the diagnosis and management of BOS were published as a combined effort by three medical professional societies [84]. As the understanding of patterns and heterogeneity within BOS and the pathophysiology of lung allograft dysfunction evolved over time, the Pulmonary Council of the ISHLT published a consensus report in 2019 proposing the term chronic lung allograft dysfunction as an umbrella term to describe the clinical range of pathologic processes occurring in the lung allograft leading to persistent lung function decline in lung transplant recipients [85]. Briefly, CLAD is defined by a decrease of $\geq 20 \%$ in $\mathrm{FEV}_{1}$ for $>3$ months from post-transplant baseline, after exclusion of other potential causes [85]. The post-transplant baseline value is calculated from the mean of the two best postoperative $\mathrm{FEV}_{1}$ measurements taken more than 3 weeks apart [85]. The etiology of CLAD is probably multifactorial, its underlying mechanisms are complex, and much of its pathophysiology remains unknown [86]. The recent advances in the understanding of the pathophysiological mechanisms of CLAD and its different phenotypes were just reviewed in detail by Verleden and colleagues [86]. In CLAD-BOS, the most common clinical CLAD phenotype, persistent micro-injuries caused by gastroesophageal reflux, viruses, microbes, etc., to the lung graft epithelium seem to result in chronic airway inflammation and aberrant wound healing with fibroblast activation and migration, eventually leading to small airway obliteration [86]. To date, no well-proven therapeutic approach is available to successfully treat CLAD in lung transplant recipients, either adults or children, according to a recent systematic review by Benden and colleagues [87]. Treatment approaches include a change/augmentation of immunosuppression, and use of neo-macrolides, extracorporeal photopheresis, and total lymphoid irradiation, but no randomized controlled trials have been conducted applying any of these therapies [87]. Several therapies have been or are being investigated for targeting CLAD-BOS after lung transplantation, including as aerosolized liposomal cyclosporine [88], alemtuzumab, a monoclonal antibody directed against CD52 [89], and antifibrotic drugs (pirfenidone, nintedanib) [90]. In the future, novel therapies are urgently needed, in particular new therapeutic approaches that not only slow or stabilize lung function decline but also reverse the disease process itself. Until then, CLAD remains the Achilles' heel of lung transplantation. 


\section{THE POTENTIAL ROLE OF CFTR MODULATORS POST LUNG TRANSPLANTATION}

With the substantial effectiveness achieved by CFTR modulator therapies with regard to CF lung disease and its impact on referral, listing, and lung transplant procedures, the focus of CFTR modulator use shifts to their impact on extrapulmonary disease manifestations of $\mathrm{CF}$ as a multisystem disorder. Thus, there is potentially a role for CFTR modulators even post lung transplantation, as extrapulmonary comorbidities of CF remain an even greater issue after successful lung transplantation, often with a substantial impact on individuals' health-related quality of life. However, it is beyond the scope of this review to evaluate the role of CFTR modulators in non-lung transplant recipients with CF.

It is important to note that primary outcomes of CFTR modulator clinical studies have focused on lung function improvement for licensing issues; however, CFTR modulators are systemic therapies affecting tissues and organs where CFTR is expressed. Positive effects of CFTR modulators have been noted in CF-related sinus disease, CF-related gastrointestinal disease manifestations, and CF-related diabetes; nevertheless, to date, these effects have not been studied systematically in randomized controlled trials [91-93].

In the case of a treatment trial of CFTR modulators post lung transplantation, caution is needed concerning potential drug-drug interactions, as these drugs are metabolized through cytochrome P450 enzymes [93]. This is of particular importance in the post-transplant setting, as calcineurin inhibitors (cyclosporine, tacrolimus) are routinely prescribed as part of the standard immunosuppression therapy regime. Further, azoles are frequently used for antifungal prophylaxis and treatment. It is advisable to seek advice from a pharmacist with knowledge of the pharmacology of CFTR modulators and potential drug interactions. It is critically important to prevent subtherapeutic immunosuppressant drug levels on the one hand, and increased risk of opportunistic infection or nephrotoxicity due to over-immunosuppression on the other hand. Prescribers have to follow indications and restrictions for CFTR modulator use in solid organ transplant recipients as set by medical licensing agencies and their countries' reimbursement policies, as CFTR modulators are expensive and reimbursement is limited in some countries.

Hayes et al. very recently published a case series of five lung transplant recipients with CF who were started on CFTR modulator therapy [94]. All patients received induction therapy with basiliximab and standard triple immunosuppression including tacrolimus, mycophenolate, and steroids. Three patients were started on tezacaftor/ivacaftor pre-transplant and continued on dual CFTR modulator treatment after transplantation; the other two patients were placed on elexacaftor/tezacaftor/ivacaftor post transplant [94]. The authors of the case series describe the extrapulmonary benefits in each individual patient, including predominantly improvement in nutritional status and reduced symptoms of CF-related chronic sinus disease [94]. Only one of the five lung transplant recipients demonstrated mildly elevated live enzymes within 1 week of starting triple CFTR modulator therapy (elexacaftor/tezacaftor/ivacaftor) [94]. The authors point out the safe usage of CFTR modulators in their small patient cohort and the extrapulmonary benefits seen; however, Hayes et al. also highlight disadvantages of the CFTR modulator usage post lung transplantation including drug-drug interactions, the need for drug dosage adjustments, and treatment costs [94]. Ramos et al., in a recently published review article, discuss additional potential benefits of CFTR modulator therapy following lung transplantation [95]. The authors focus on the multifactorial development of CLAD post lung transplant and the extrapulmonary manifestations of CF that potentially contribute to CLAD development such as gastroesophageal reflux disease and CFassociated chronic sinus disease [95]. Further, as there is evidence that the CFTR protein works as a tumor suppressor, improvement in CFTR function could potentially reduce the risk of malignancies following lung transplantation 
[95]. Based on the current published literature, a recent CF Foundation consensus statement found a lack of evidence to recommend for or against the use of CFTR modulators in patients after lung transplantation [78]. There remains a need for clinical studies to assess the safety and efficacy of CFTR modulator therapy in CF lung transplant recipients.

Finally, organ allocation practices may need to be readdressed in the future taking into consideration the clinical impact of highly effective new CFTR modulator therapies in lung transplant candidates with CF. Given that organ allocation practices are governed not only by medical and bioethical necessities, but also by traditions, legislation, and practical circumstances that vary substantially across the world [96], this aspect is well beyond the scope of this review article.

\section{CONCLUSIONS}

Overall, survival in individuals with CF has improved substantially in recent years, even before treating the underlying defect in the gene encoding the CFTR protein. Despite the fact that $\mathrm{CF}$ is a multisystem disorder affecting tissues and organs where CFTR is expressed, CF lung disease causes most of the morbidity and mortality. At the present time, lung transplantation is considered the ultimate therapeutic option for individuals with progressive ACFLD. Since CFTR modulators have become available, these drugs are now being used in selected individuals even with ACFLD and have shown promising results; however, randomized controlled trial data on CFTR modulators in this subgroup of patients are lacking. Therefore, further randomized controlled trials in patients with ACFLD are needed. And ultimately, new consensus guidelines for the selection of $\mathrm{CF}$ candidates for lung transplantation should take the new CFTR modulator therapies into consideration regarding timing of referral and timing of listing for transplant.

\section{ACKNOWLEDGEMENTS}

Funding. No funding or sponsorship was received for this study or publication of this article.

Authorship. All named authors meet the International Committee of Medical Journal Editors (ICMJE) criteria for authorship for this article, take responsibility for the integrity of the work as a whole, and have given their approval for this version to be published.

Author contributions. Both named authors contributed equally to the concept, design, and drafting of the manuscript.

Disclosures. Christian Benden has received honorary speaker fees from Vertex Pharmaceuticals and Mallinckrodt Pharmaceuticals, and has served on a scientific advisory board to Zambon Pharma. Carsten Schwarz has received honorary speaker fees and advisory board fees from AbbVie, Chiesi Farmaceutici, Horizon Therapeutics, Mylan Pharmaceuticals, Novartis, Proteostasis Therapeutics, and Vertex Pharmaceuticals.

Compliance with ethics guidelines. This article is based on reviews of current consensus statements and published literature, and does not involve any studies with human participants or animals conducted by any of the authors.

Open Access. This article is licensed under a Creative Commons Attribution-NonCommercial 4.0 International License, which permits any non-commercial use, sharing, adaptation, distribution and reproduction in any medium or format, as long as you give appropriate credit to the original author(s) and the source, provide a link to the Creative Commons licence, and indicate if changes were made. The images or other third party material in this article are included in the article's Creative Commons licence, unless indicated otherwise in a credit line to the material. If material is not included in the article's Creative Commons licence and 
your intended use is not permitted by statutory regulation or exceeds the permitted use, you will need to obtain permission directly from the copyright holder. To view a copy of this licence, visit http://creativecommons.org/licenses/by$\mathrm{nc} / 4.0 /$.

\section{REFERENCES}

1. Bosch B, De Boeck K. Searching for a cure for cystic fibrosis. A 25-year quest in a nutshell. Eur J Pediatr. 2016;175:1-8.

2. De Boeck K. Cystic fibrosis in the year 2020: a disease with a new face. Acta Paediatr. 2020;109: 893-9.

3. Fajac I, De Boeck K. New horizons for cystic fibrosis treatment. Pharmacol Ther. 2017;170:205-11.

4. Benden C. Lung transplantation as standard of care for advanced cystic fibrosis lung disease. J Heart Lung Transpl. 2020;39:561-2.

5. Kapnadak SG, Dimango E, Hadjiliadis D, et al. Cystic Fibrosis Foundation consensus guidelines for the care of individuals with advanced cystic fibrosis lung disease. J Cyst Fibros. 2020;19:344-54.

6. Weill D, Benden C, Corris PA, et al. A consensus document for the selection of lung transplant candidates: 2014 an update from the Pulmonary Transplantation Council of the International Society for Heart and Lung Transplantation. J Heart Lung Transpl. 2015;34:1-15.

7. Morrell MR, Kiel SC, Pilewski JM. Organ transplantation for cystic fibrosis. Semin Respir Crit Care Med. 2019;40:842-56.

8. Chambers DC, Cherikh WS, Harhay MO, et al. The International Thoracic Organ Transplant Registry of the International Society for Heart and Lung Transplantation: thirty-sixth adult lung and heartlung transplantation report 2019; focus theme: donor and recipient size match. J Heart Lung Transpl. 2019;38:1042-55.

9. De Boeck K, Amaral MD. Progress in therapies for cystic fibrosis. Lancet Respir Med. 2016;4:662-74.

10. Bell SC, Mall MA, Gutierrez H, et al. The future of cystic fibrosis care: a global perspective. Lancet Respir Med. 2020;8:65-124.

11. Elborn JS. Cystic fibrosis. Lancet. 2016;388: 2519-31.
12. Cystic Fibrosis Foundation. Cystic Fibrosis Foundation Patient Registry 2019 Annual Data Report. Bethesda, Maryland Cystic Fibrosis Foundation. 2020.

13. Cystic Fibrosis Trust. UK Cystic Fibrosis Registry 2018 Annual Data Report. 2019.

14. Ratjen F, Bell SC, Rowe SM, et al. Cystic fibrosis. Nat Dis Primers. 2015;1:15010.

15. Rowe SM, Miller S, Sorscher EJ. Cystic fibrosis. N Engl J Med. 2005;352:1992-2001.

16. Zielenski J, Tsui LC. Cystic fibrosis: genotypic and phenotypic variations. Ann Rev Genet. 1995;29: 777-807.

17. Yang Y, Devor DC, Engelhardt JF, et al. Molecular basis of defective anion trans- port in L cells expressing recombinant forms of CFTR. Hum Mol Genet. 1993;2:1253-61.

18. Van Goor F, Hadida S, Grootenhuis PD, et al. Rescue of CF airway epithelial cell function in vitro by a CFTR potentiator, VX-770. Proc Natl Acad Sci USA. 2009;106:18825-30.

19. Accurso FJ, Rowe SM, Clancy JP, et al. Effect of VX770 in persons with cystic fibrosis and the G551DCFTR mutation. N Engl J Med. 2010;363: 1991-2003.

20. Ramsey BW, Davies J, McElvaney NG, et al. A CFTR potentiator in patients with cystic fibrosis and the G551D mutation. N Engl J Med. 2011;365:1663-72.

21. Du K, Sharma M, Lukacs GL. The $\Delta$ F508 cystic fibrosis mutation im- pairs domain-domain interactions and arrests post-translational folding of CFTR. Nat Struct Mol Biol. 2005;12:17-25.

22. Du K, Lukacs GL. Cooperative assembly and misfolding of CFTR domains in vivo. Mol Biol Cell. 2009;20:1903-15.

23. Yu H, Burton B, Huang CJ, et al. Ivacaftor potentiation of multiple CFTR channels with gating mutations. J Cyst Fibros. 2012;11:237-45.

24. Phuan PW, Veit G, Tan JA, Finkbeiner WE, Lukacs GL, Verkman AS. Potentiators of defective $\Delta$ F508CFTR gating that do not interfere with corrector action. Mol Pharmacol. 2015;88:791-9.

25. Park J, Khloya P, Seo Y, et al. Potentiaton of $\Delta$ F508and G551D-CFTR-mediated Cl- current by novel hydroxypyrazolines. PLoS ONE. 2016;11: e0149131.

26. Gees M, Much S, Van der Plas S, et al. Identification and characterization of novel CFTR potentiators. Front Pharmacol. 2018;9:1221. 
27. Harbeson SL, Morgan AJ, Liu JF, et al. Altering metabolic profiles of drugs by precision deuteration 2: discovery of a deuterated analog of ivacaftor with differentiated pharmacokinetics for clinical development. J Pharmacol Exp Ther. 2017;362:359-67.

28. Okiyoneda T, Veit G, Dekkers JF, et al. Mechanismbased corrector combination restores $\triangle \mathrm{F} 508$-CFTR folding and function. Nat Chem Biol. 2013;9: 444-54.

29. Wainwright CE, Elborn JS, Ramsey BW, et al. Lumacaftor-ivacaftor in patients with cystic fibrosis homozygous for phe508del CFTR. N Eng J Med. 2015;373:220-31.

30. Taylor-Cousar JL, Munck A, McKone EF, et al. Tezacaftor-Ivacaftor in patients with cystic fibrosis homozygous for Phe508del. N Eng J Med. 2017;377: 2013-23.

31. Donaldson SH, Pilewski JM, Griese M, et al. Tezacaftor/Ivacaftor in subjects with cystic fibrosis and F508del/F508del-CFTR or F508del/G551D-CFTR. Am J Respir Crit Care Med. 2018;197:214-24.

32. Rowe SM, Daines C, Ringshausen FC, et al. Tezacaftor-Ivacaftor in residual-function heterozygotes with cystic fibrosis. N Eng J Med. 2017;377: 2024-34.

33. Davies JC, Moskowitz SM, Brown C, et al. VX-659tezacaftor-ivacaftor in patients with cystic fibrosis and one or two phe508del alleles. N Eng J Med. 2018;379:1599-611.

34. Keating D, Marigowda G, Burr L, et al. VX-445tezacaftor-ivacaftor in patients with cystic fibrosis and one or two phe508del alleles. N Engl J Med. 2018;379:1612-20.

35. Middleton PG, Mall MA, Drevínek P, et al. Elexacaftor-tezacaftor-ivacaftor for cystic fibrosis with a single phe508del allele. N Eng J Med. 2019;381: 1809-19.

36. Heijerman HGM, McKone EF, Downey DG, et al. Efficacy and safety of the elexacaftor plus tezacaftor plus ivacaftor combination regimen in people with cystic fibrosis homozygous for the F508del mutation: a double-blind, randomised, phase 3 trial. Lancet. 2019;394:1940-8.

37. The Association of the Scientific Medical Societies in Germany. Registered guideline project. CFTRmodulator therapy in cystic fibrosis - living guideline. https://www.awmf.org/leitlinien/detail/ anmeldung/1/11/020-026.html. Accessed 01 July 2020.

38. Arooj P, Morrissy D, Ronan N, et al. Real-world orkambi cohort CorK study (ROCK): a prospective twelve months' analysis addressing the effectiveness of CFTR modulation in patients with cystic fibrosis homozygous for F508del CFTR. J Cyst Fibros. 2019;18:S131-2.

39. Burgel P, Munck A, Durieu I, et al. Real-life safety and effectiveness of lumacaftor-ivacaftor in patients with cystic fibrosis. Am J Respir Crit Care Med. 2020;201:188-97.

40. Gomez-Pastrana D, Nwokoro C, McLean M, et al. Real-world effectiveness of ivacaftor in children with cystic fibrosis and the G551D mutation. An Pediatr. 2019;90:148-56.

41. Higgins M, Volkova N, Moy K, et al. Real-world outcomes among patients with cystic fibrosis treated with ivacaftor: 2012-2016 experience. Pulm Ther. 2020;6:141-9.

42. Volkova N, Moy K, Evans J, et al. Disease progression in patients with cystic fibrosis treated with ivacaftor: data from national US and UK registries. J Cyst Fibros. 2020;19:68-79.

43. Lopes-Pacheco M. CFTR modulators: the changing face of cystic fibrosis in the era of precision medicine. Front Pharmacol. 2020;10:1662.

44. Cystic Fibrosis Foundation. Drug development pipeline. https://www.cff.org/Trials/pipeline. Accessed 01 July 2020.

45. McCague AF, Raraigh KS, Pellicore MJ, et al. Correlating cystic fibrosis transmembrane conductance regulator function with clinical features to inform precision treatment of cystic fibrosis. Am J Respir Crit Care Med. 2019;199:1116-26.

46. De Winter-de Groot KM, Janssens HM, van Uum RT, et al. Stratifying infants with cystic fibrosis for disease severity using intestinal organoids swelling as a biomarker of CFTR function. Eur Respir J. 2018;52:1702529.

47. Barry PJ, Plant BJ, Nair A, et al. Effects of ivacaftor in patients with cystic fibrosis who carry the G551D mutation and have severe lung disease. Chest. 2014;146:152-8.

48. Salvatore D, Carnovale V, Iacotucci P, et al. Effectivenesss of ivacaftor in severe cystic fibrosis patients and non-G551D gating mutations. Pediatr Pulmonol. 2019;54:1398-403.

49. Elborn JS, Ramsey BW, Boyle MP, et al. Efficacy and safety of lumacaftor/ivacaftor combination therapy in patients with cystic fibrosis homozygous for Phe508del CFTR by pulmonary function subgroup: a pooled analysis. VX-809 TRAFFIC and TRANSPORT study groups. Lancet Respir Med. 2016;4: 617-26. 
50. Ejiofor LCK, Mathiesen IHM, Jensen-Fangel S, et al. Patients with cystic fibrosis and advanced lung disease benefit from lumacaftor/ivacaftor treatment. Pediatr Pulmonol. 2020;55:3364-70.

51. Hubert D, Chiron R, Camara B, et al. Real-life initiation of lumacaftor/ivacaftor combination in adults with cystic fibrosis homozygous for the Phe508del CFTR mutation and severe lung disease. J Cyst Fibros. 2017;16:388-91.

52. Popowicz N, Wood J, Tai A, et al. Immediate effects of lumacaftor/ivacaftor administration on lung function in patients with severe cystic fibrosis lung disease. J Cyst Fibros. 2017;16:392-4.

53. Taylor-Cousar J, Jain M, Barto TL, et al. Lumacaftor/ ivacaftor in patients with cystic fibrosis and advanced lung disease homozygous for F508delCFTR. J Cyst Fibros. 2018;17:228-35.

54. Tong K, Barker D, France M, et al. Lumacaftor/ivacaftor reduces exacerbations in adults homozygous for Phe508del mutation with severe lung disease. J Cyst Fibros. 2020;19:415-20.

55. King SJ, Keating D, Williams E, Paul E, Borg BM, Finlayson F, Button BM, Wilson JW, Kotsimbos T. Lumacaftor/ivacaftor-associated health stabilisation in adults with severe cystic fibrosis. ERJ Open Res. 2021;7:00203-2020.

56. Murer C, Huber LC, Kurowski T, et al. First experience in Switzerland in Phe508del homozygous cystic fibrosis patients with end-stage pulmonary disease enrolled in a lumacaftor-ivacaftor therapy trial - preliminary results. Swiss Med Wkly. 2018;148: w14593.

57. Schwarz C, Sutharsan S, Epaud R, et al. Tezacaftor/ ivacaftor in people with cystic fibrosis who stopped lumacaftor/ivacaftor due to respiratory adverse events. J Cyst Fibros. 2021;20:228-33.

58. O'Shea KM, O'Carroll OM, Caroll C, et al. Efficacy of elexacaftor/tezacaftor/ivacaftor in patients with cystic fibrosis and advanced lung disease. Eur Respir J. 2021;57:2003079.

59. Patterson GA, Cooper JD, Goldman B, et al. Technique of successful clinical double-lung transplantation. Ann Thorac Surg. 1988;45:626-33.

60. Benoit TM, Benden C. Pediatric lung transplantation: supply and demand. Curr Opin Organ Transpl. 2019;24:324-8.

61. Hayes D Jr, Benden C, Sweet SC, et al. Current state of pediatric lung transplantation. Lung. 2015;193: 629-37.
62. Benden C. Pediatric lung transplantation. J Thorac Dis. 2017;9:2675-83.

63. Werner R, Benden C. Pediatric lung transplantation as standard of care. Clin Transpl. 2021;35: e14126.

64. Hayes D Jr, Cherikh WS, Chambers DC, et al. The International Thoracic Organ Transplant Registry of the International Society for Heart and Lung Transplantation: twenty-second pediatric lung and heart-lung transplantation report 2019; focus theme: donor and recipient size match. J Heart Lung Transpl. 2019;38:1015-27.

65. Lay C, Law N, Holm AM, Benden C, Aslam S. Outcomes in cystic fibrosis lung transplant recipients infected with organisms labeled as pan-resistant: an ISHLT registry-based analysis. J Heart Lung Transpl. 2019;38:545-52.

66. Aigner C, Wisser W, Taghavi S, et al. Institutional experience with extracorporeal membrane oxygenation in lung transplantation. Eur J Cardiothorac Surg. 2007;31:468-73.

67. Cypel M, Keshavjee S. Extracorporeal life support as a bridge to lung transplantation. Clin Chest Med. 2011;32:245-51.

68. Hoopes CW, Kukreja J, Golden J, et al. Extracorporeal membrane oxygenation as bridge to pulmonary transplantation. J Thorac Cardiovasc Surg. 2013;145:862-7.

69. Inci I, Klinzing S, Schneiter D, et al. Outcome of extracorporeal membrane oxygenation as a bridge to lung transplant: an institutional experience and literature review. Transplantation. 2015;99: 1667-71.

70. Schmid FA, Benden C. Special considerations for the use of lung transplantation in pediatrics. Expert Rev Respir Med. 2016;10:655-62.

71. Schmid F, Sasse M, Boehne M, et al. Concept of «awake veno-venous extracorporeal membrane oxygenation" in pediatric patients awaiting lung transplantation. Pediatr Transpl. 2013;17:224-30.

72. Abrams D, Brodie D, Arcasoy SM. Extracorporeal life support in lung transplantation. Clin Chest Med. 2017;38:655-66.

73. Lancaster TS, Eghtesady P. State of the art in pediatric lung transplantation. Semin Thorac Cardiovasc Surg. 2018;30:166-74.

74. Ramos KJ, Smith PJ, McKone EF, et al. Lung transplant referral for individuals with cystic fibrosis: Cystic Fibrosis Foundation consensus guidelines. J Cyst Fibros. 2019;18:321-33. 
75. Bermingham B, Rueschhoff A, Ratti G, et al. Shortterm effect of elexacaftor-tezacaftor-ivacaftor on lung function and transplant planning in cystic fibrosis patients with advanced lung disease. J Cyst Fibros. 2021.

76. Shteinberg M, Taylor-Cousar JL. Impact of CFTR modulator use on outcomes in people with severe cystic fibrosis. Eur Respir Rev. 2020;29:190112.

77. Southern KW, Murphy J, Sinha IP, Nevitt SJ. A systematic Cochrane review of corrector therapies (with or without potentiators) for people with cystic fibrosis with class II gene variants (most commonly F508DEL). Paediatr Respir Rev. 2021;38: $25-8$.

78. Shah P, Lowery E, Chaparro C, et al. Cystic Fibrosis Foundation consensus statement for the care of cystic fibrosis lung transplant recipients. J Heart Lung Transpl. 2021;40:539-56.

79. Hayes D Jr, Sweet SC, Benden C, et al. Transplant center volume and outcomes in lung transplantation for cystic fibrosis. Transpl Int. 2017;30:371-7.

80. Paraskeva MA, Edwards LB, Levvey B, et al. Outcomes of adolescent recipients after lung transplantation: an analysis of the International Society for Heart and Lung Transplantation Registry. J Heart Lung Transpl. 2018;37:323-31.

81. Goldfarb SB, Hayes D Jr, Levvey BJ, et al. The International Thoracic Organ Transplant Registry of the International Society for Heart and Lung Transplantation: twenty-first pediatric lung and heart-lung transplantation report 2018; focus theme: multiorgan transplantation. J Heart Lung Transpl. 2018;37:1196-206.

82. Benden C, Goldfarb SB, Stehlik J. An aging population of patients with cystic fibrosis undergoes lung transplantation: an analysis of the ISHLT Thoracic Transplant Registry. J Heart Lung Transpl. 2019;38:1162-9.

83. Burgel PR, Durieu I, Chiron R, et al. Rapid improvement after starting elexacaftor-tezacaftorivacaftor in patients with cystic fibrosis and advanced pulmonary disease. Am J Respir Crit Care Med. 2021;204:64-73.

84. Meyer KC, Raghu G, Verleden GM, et al. An international ISHLT/ATS/ERS clinical practice guideline: diagnosis and management of bronchiolitis obliterans syndrome. Eur Respir J. 2014;44:1479-503.

85. Verleden GM, Glanville AR, Lease ED, et al. Chronic lung allograft dysfunction: definition, diagnostic criteria, and approaches to treatment: a consensus report from the Pulmonary Council of the ISHLT. J Heart Lung Transpl. 2019;38:493-503.

86. Verleden SE, Von der Thüsen J, Roux A, et al. When tissue is the issue: a histological review of chronic lung allograft dysfunction. Am J Transpl. 2020;20: 2644-51.

87. Benden C, Haughton M, Leonard S, Huber LC. Therapy options for chronic lung allograft dysfunction bronchiolitis obliterans syndrome following first-line immunosuppression strategies: a systematic review. J Heart Lung Transpl. 2017;36: 921-33.

88. Iacono A, Wijesinha $\mathrm{M}$, Rajagopal $\mathrm{K}$, et al. A randomised single-centre trial of inhaled liposomal cyclosporine for bronchiolitis obliterans syndrome post-lung transplantation. ERJ Open Res. 2019;5: 00167-2019.

89. Moniodis A, Townsend K, Rabin A, et al. Comparison of extracorporeal photopheresis and alemtuzumab for the treatment of chronic lung allograft dysfunction. J Heart Lung Transpl. 2018;37:340-8.

90. Von Suesskind-Schwendi M, Heigel E, Pfaehler S, et al. Protective function of pirfenidone and everolimus on the development of chronic allograft rejection after experimental lung transplantation. Histol Histopathol. 2016;31:793-805.

91. Bellin MD, Laguna T, Leschyshyn J, et al. Insulin secretion improves in cystic fibrosis following ivacaftor correction of CFTR: a small pilot study. Pediatr Diabetes. 2013;14:417-21.

92. Sheikh SI, Long FR, McCoy KS, Johnson T, RyanWenger NA, Hayes D Jr. Ivacaftor improves appearance of sinus disease on computerized tomography in cystic fibrosis patients with G551 mutation. Clin Otolaryngol. 2015;40:16-21.

93. Mitchell RM, Jones AM, Barry PJ. CFTR modulator therapy in patients with cystic fibrosis and an organ transplant. Paediatr Respir Rev. 2018;27:6-8.

94. Hayes D Jr, Darland LK, Hjelm MA, Mansour HM, Wikenheiser-Brokamp KA. To treat ot not to treat: CFTR modulators after lung transplantation. Pediatr Transpl. 2021;25:e14007.

95. Ramos KJ, Pilewski JM, Taylor-Cousar JL. Challenges in the use of highly effective modulator treatment for cystic fibrosis. J Cyst Fibros. 2021;20: $381-7$.

96. Holm AM, Immer F, Benden C. Lung allocation for transplant: the European perspective. Clin Transpl. 2020;34: e13883. 